\title{
Aislamiento e identificación de metabolitos secundarios mayoritarios presentes en el extracto metanólico de Sapium lateriflorum (Euphorbiaceae)
}

JONATHAN VERDEZOTO a, *
a Universidad Central del Ecuador

*Correspondencia: jonatvv@hotmail.com

Recibido: 24 febrero 2012 / Aceptado: 28 mayo 2012

\section{Resumen}

La especie Sapium lateriflorum Hemsl, fue considerada para esta investigación debido al escaso conocimiento que se tiene de su composición química y del género al cual pertenece.

Al extracto metanólico, obtenido de las partes aéreas (ramas y hojas) de Sapium lateriflorum, se le realizó una separación líquido-líquido, con diclorometano y con una solución de metanol-agua (65:35). A cada una de las dos fases obtenidas de la partición, se fraccionó posteriormente mediante cromatografía en columna. Los compuestos aislados, una vez purificados, se identificaron mediante sus características físicas y espectroscópicas. Los compuestos obtenidos de la fase orgánica (diclorometano), son: sitostenona, lupeol, $\beta$-sitosterol, vomifoliol, y un ácido graso.

Palabras clave: Sapium lateriflorum, sitostenona, $\beta$-sitosterol, lupeol, vomifoliol, Euphorbiaceae.

\section{Isolation and Identification of the majority secondary metabolites present in methanolic extract of Sapium lateriflorum (Euphorbiaceae)}

\section{Abstract}

The species Sapium lateriflorum Hemsl., was considered for this research, due to the scanty knowledge about the chemical composition from this species and the genus to which it belongs.

The methanolic extract from the aerial parts (branches and leaves) of Sapim lateriflorum, was subjected to liquidliquid separation, with dichloromethane and a solution of methanol-water solution (65: 35). Each one of the phases obtained from the partition was purified through column chromatography. Compounds purified from the organic phase (dichloromethane), were identified spectroscopically as sitostenone, lupeol, $\beta$-sitosterol, vomifoliol, and a fatty acid.

Key words: Sapium lateriflorum, sitostenone, $\beta$-sitosterol, lupeol, vomifoliol, Euphorbiaceae. 


\section{Introducción}

El conocimiento de los componentes naturales de las plantas resulta ser de gran interés medicinal, agrícola y alimentario, desde que la persona ha hecho uso de ellas para cubrir algunas de sus necesidades vitales, esto hace del estudio de plantas, un valioso aporte a los conocimientos que sobre ellas se han venido acumulando a lo largo del tiempo, desde que las utilizaron los pueblos ancestrales.

Las especies vegetales incluidas en la familia Euphorbiaceae tienen alto aprecio popular, ya que entre ellas se encuentran plantas ornamentales (como la nochebuena), medicinales (como el ricino), comestibles (como la chaya), entre otras. Esta familia ha resultado ser fuente promisoria de metabolitos con propiedades medicinales. La composición fitoquímica de las diversas especies de Euphorbiaceae es poco conocida, y debido a que se han encontrado metabolitos de importancia farmacológica, estas plantas ameritan ser analizadas con dedicación; siendo el estudio de Sapium lateriflorum, un gran aporte al conocimiento científico de las especies correspondientes al grupo de las euforbiáceas.

El género Sapium, es un grupo que se ha estudiado poco y en estudios previos, se han aislado derivados de forbol que en algunos casos han demostrado ser bioactivos [1, 2].

Análisis químicos previos sobre Sapium lateriflorum indican que el lupeol (2) y el vomifoliol (5) son substancias que se encuentran presentes en los extractos de menor polaridad según el Instituto de Química de la Universidad Nacional Autónoma de México, Laboratorio 1C.

El interés de aislar e identificar los metabolitos secundarios mayoritarios presentes en el extracto metanólico de las partes aéreas (hojas y ramas) de Sapium lateriflorum (Euphorbiaceae), surge del conocimiento creciente de que muchos de los metabolitos secundarios de organismos, incluyendo plantas, cumplen importantes funciones biológicas y ecológicas, principalmente, como mensajeros químicos y compuestos de defensa $[3,4]$.

\section{Parte experimental}

\section{Población y muestra}

El extracto metanólico seco, usado en la presente investigación, está disponible en el laboratorio $1 \mathrm{C}$ del Instituto de Química de la UNAM y proviene del seca- do a la sombra, a temperatura ambiente, y trituración de las partes aéreas de la planta recolectada el año 2007 en la localidad de "San Juan Chancalaíto", que está situada en el Municipio de Palenque (Estado de Chiapas), México. La planta se identificó con el código MEXU 39267 por el biólogo Esteban Manuel Martínez Salas, del Instituto de Biología de la Universidad Nacional Autónoma de México y está depositada en el Herbario Nacional de la UNAM.

El extracto fue preparado a partir de $1.1 \mathrm{Kg}$ de hojas y ramas secas. Después de triturar el material, se sometió a maceración con hexano durante 24 horas por dos veces consecutivas. Del extracto recuperado por filtración, se evaporó el disolvente a presión reducida y calentamiento suave. Después de eliminar el hexano del residuo vegetal, se adicionó acetato de etilo y se repitió el procedimiento de filtración y eliminación del disolvente. Dicho residuo se maceró nuevamente con metanol, siguiendo el mismo procedimiento. Finalmente, se obtuvieron $45.6 \mathrm{~g}$ de extracto hexánico seco; $17.34 \mathrm{~g}$ de extracto de acetato de etilo seco y $28.1 \mathrm{~g}$ de extracto metanólico seco.

\section{Técnicas e instrumentos analíticos}

Las destilaciones al vacío se hicieron en sistemas evaporadores rotatorios de marca BUCHI. El aparato empleado para determinar los puntos de fusión fue Cole-Parmer y las mediciones no están corregidas. Las muestras fueron identificadas mediante los espectros de $\mathrm{RMN}$ de ${ }^{1} \mathrm{H}$ y de ${ }^{13} \mathrm{C}$ obtenidos utilizando los espectrómetros Varian Unity 300 (300/75 MHz), Bruker Avance III (400/100MHz) y Varian Unity Plus (500/125 MHz). Todos los espectros fueron tomados a $20^{\circ} \mathrm{C}$ utilizando como disolvente cloroformo deuterado $\left(\mathrm{CDCl}_{3}\right)$ y tetrametilsilano (TMS) como estándar interno; los desplazamiento químicos (ठ) están expresados en partes por millón (ppm) y las constantes de acoplamiento (J) se reportan en Hertz $(\mathrm{Hz})$.

Las cromatografías en capa fina (CCF) se realizaron en placas de aluminio recubiertas de gel de sílice (Merck $60 \mathrm{~F}_{254}$ ). Las cromatografías en columna fueron desarrolladas usando gel de sílice (malla 230-400). Los eluyentes utilizados estaban conformados por disolventes orgánicos puros y mezclas en proporciones adecuadas de: hexano, diclorometano, acetato de etilo, acetona, metanol. La visualización de los compuestos en las placas CCF se llevó a cabo bajo lám- 
paras SPECTROLINE de luz UV de onda corta y onda larga (254 nm y $365 \mathrm{~nm}$ ); posteriormente, las placas se revelaron químicamente utilizando sulfato cérico amoniacal con calentamiento, realizado con ayuda de una secadora industrial. Los espectros de masas se obtuvieron en espectrómetros JEOL-AX505HA.

\subsubsection{Partición del extracto}

El extracto metanólico seco, de las hojas y ramas de Sapium lateriflorum (28.1 g ya disponibles en el laboratorio), se disolvió en metanol y se extrajo con diclorometano (fase orgánica). Para esto se disminuyó la miscibilidad del metanol agregando agua hasta obtener una relación metanol/agua 65:35.

Después de hacer pruebas de separación mediante $C C F$, se determinó que la fase hidro-alcohólica no era apropiada como tal, por lo que se derivatizó una porción de dicha fase con la intención de mejorar su separación cromatográfica al disminuir la polaridad de grupos hidroxilo de los compuestos presentes, utilizando anhídrido acético en piridina, obteniéndose 1.5 gramos de porción acetilada.

\subsubsection{Elucidación estructural}

Las estructuras fueron finalmente confirmadas por sus espectros y propiedades físicas, comparándolas con muestras de referencia disponibles en el laboratorio. Las asignaciones de los desplazamientos químicos se hicieron por comparación con los datos previamente reportados y las reglas de desplazamiento químico.

Sitostenona (1) con pf $96-98^{\circ} \mathrm{C}$ (lit p.f. $97-99{ }^{\circ} \mathrm{C}$ [5]); $\mathbf{R f}$ (Hex:AcOEt 8:2) = 0.60; EM m/z (\% intensidad relativa): $412\left(M^{+}, 40\right), 397(9), 370(12), 288(19), 271(12)$, 229(35), 187(8), 173(9), 147(25), 124(100); RMN-'H $(400 \mathrm{MHz}, \mathrm{CDCl} 3) \delta 5.72(1 \mathrm{H}, \mathrm{s}, \mathrm{H}-4), 2.33(4 \mathrm{H}, \mathrm{m}$, $\mathrm{H}-2, \mathrm{H}-6), 2.08-1.98(3 \mathrm{H}, \mathrm{m}, \mathrm{H}-1, \mathrm{H}-12), 1.88-1.82$ $(2 \mathrm{H}, \mathrm{m}, \mathrm{H}-7, \mathrm{H}-15), 1.18(3 \mathrm{H}, \mathrm{s}, \mathrm{H}-19), 0.92(3 \mathrm{H}, \mathrm{d}, \mathrm{J}$ $=6.6 \mathrm{~Hz}, \mathrm{H}-21), 0.84(3 \mathrm{H}, \mathrm{d}, J=7.1 \mathrm{~Hz}, \mathrm{H}-26), 0.81$ $(3 \mathrm{H}, \mathrm{s}, \mathrm{J}=6.9 \mathrm{~Hz}, \mathrm{H}-27), 0.71(3 \mathrm{H}, \mathrm{s}, \mathrm{H}-18) ; \mathrm{RMN}^{-13} \mathrm{C}$ (101 MHz, CDCl $)_{3}$ ) $\mathrm{c} 36.27$ (C-1), 34.03 (C-2), 199.85 (C-3), 123.90 (C-4), 171.90 (C-5), 34.15 (C-6), 32.20 (C-7), 35.78 (C-8), 53.97 (C-9), 38.76 (C-10), 19.97 (C11), 39.78 (C-12), 42.54 (C-13), 56.03 (C-14), 24.34 (C-15), 28.35 (C-16), 56.15 (C-17), 12.12 (C-18), 21.18 (C-19), 35.84 (C-20), 17.54 (C-21), 33.11 (C22), 26.21 (C-23), 45.98 (C-24), 29.29 (C-25), 19.17 (C-26), 18.85 (C-27), 23.22 (C-28), 12.12 (C-29).

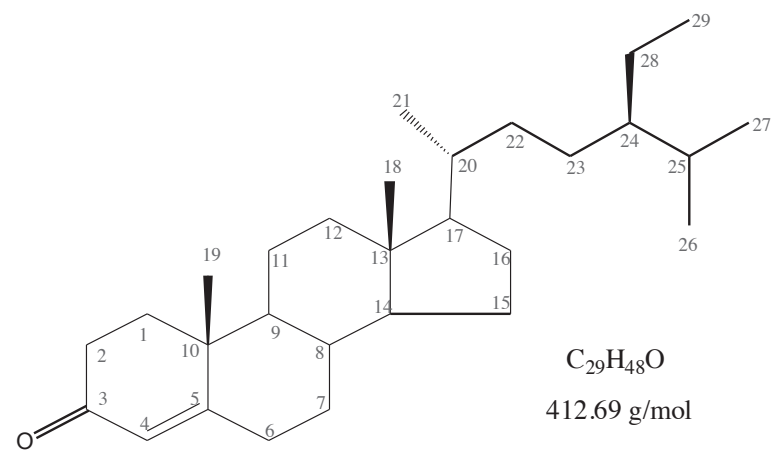

Estructura química de Sitostenona (1)

Lupeol (2) con pf 186 a $188^{\circ} \mathrm{C}$ (lit. 212-214 ${ }^{\circ} \mathrm{C}$ [6]); Rf (Hex:AcOEt 8:2) = 0.44; EM m/z (\%) 426(82), 409(23), $218(60), 207(90), 189(100) ;$ RMN-$^{-} \mathrm{H}(400$ $\left.\mathrm{MHz}_{1} \mathrm{CDCl}_{3}\right) \delta 4.69(1 \mathrm{H}, \mathrm{m}, \mathrm{Hb}-29), 4.57(1 \mathrm{H}, \mathrm{m}$, $\mathrm{Ha}-29), 3.19(1 \mathrm{H}, \mathrm{dd}, \mathrm{J}=11.2,5.0 \mathrm{~Hz}, \mathrm{H}-3), 1.68$ $(3 \mathrm{H}, \mathrm{s}, \mathrm{H}-30), 1.03(3 \mathrm{H}, \mathrm{s}, \mathrm{H}-26), 0.97(3 \mathrm{H}, \mathrm{s}, \mathrm{H}-23)$, $0.94(3 \mathrm{H}, \mathrm{s}, \mathrm{H}-27), 0.83(3 \mathrm{H}, \mathrm{s}, \mathrm{H}-25), 0.79(3 \mathrm{H}, \mathrm{s}$, $\mathrm{H}-28), 0.76(3 \mathrm{H}, \mathrm{s}, \mathrm{H}-24) ; \mathrm{RMN}^{-13} \mathrm{C}\left(101 \mathrm{MHz}, \mathrm{CDCl}_{3}\right)$ бc 38.86 (C-1), 27.59 (C-2), 79.14 (C-3), 39.01 (C4), 55.45 (C-5), 18.47 (C-6), 34.43 (C-7), 40.98 (C8), 50.59 (C-9), 37.32 (C-10), 21.08 (C-1 1), 25.29 (C-12), 38.20 (C-13), 42.98 (C-14), 27.57 (C-15), 35.73 (C-16), 43.15 (C-17), 48.13 (C-18), 48.45 (C-19), 151.11 (C-20), 30.00 (C-21), 40.15 (C-22), 28.14 (C-23), 15.52 (C-24), 16.27 (C-25), 16.13 (C-26), 14.70 (C-27), 18.15 (C-28), 109.48 (C-29), 19.46 (C-30).

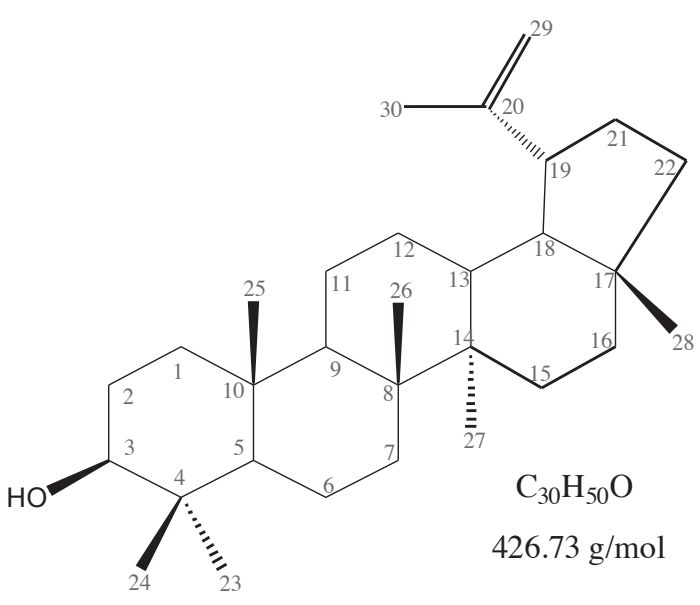

Estructura química de Lupeol (2)

$\beta$-Sitosterol (4) con pf 128 a $131^{\circ} \mathrm{C}$ (lit. 130-134 ${ }^{\circ} \mathrm{C}$ [7]); $\mathbf{R f}$ (Hex:AcOEt 8:2) = 0.28; EM (intensidad relati$\mathrm{va}, \%): \mathrm{m} / \mathrm{z} 414\left(\mathrm{M}^{+}\right)(100), 396$ (49.9), 381 (24.3), 
329 (28.0), 303 (32.3), 273 (32.7), 255 (69.3), 213 (37.9), 159 (42.9), 145 (45.1); RMN-1' $(400 \mathrm{MHz}$, CDCl3) $\delta 5.35(1 \mathrm{H}, \mathrm{d}, \mathrm{J}=5.2 \mathrm{~Hz}, \mathrm{H}-6), 3.53(1 \mathrm{H}$, $m, H-3), 1.01(3 \mathrm{H}, \mathrm{s}, \mathrm{H}-19), 0.92(3 \mathrm{H}, \mathrm{d}, \mathrm{J}=6.6 \mathrm{~Hz}$, $\mathrm{H}-21), 0.87-0.80(9 \mathrm{H}, \mathrm{m}, \mathrm{H}-26, \mathrm{H}-27, \mathrm{H}-29), 0.68$ $(3 \mathrm{H}, \mathrm{s}, \mathrm{H}-18) ; \mathrm{RMN}^{13} \mathrm{C}(101 \mathrm{MHz}, \mathrm{CDCl})$ бc 37.41 (C-1), 29.85 (C-2), 71.98 (C-3), 39.93 (C-4), 140.92 (C-5), 121.88 (C-6), 32.07 (C-7), 31.82 (C-8), 50.29 (C-9), 36.30 (C-10), 21.24 (C-11), 40.65 (C-12), 42.46 (C-13), 56.93 (C-14), 24.46 (C-15), 28.40 (C-16), 56.21 (C-17), 12.01 (C-18), 19.19 (C-19), 36.66 (C-20), 18.93 (C-21), 34.11 (C-22), 26.24 (C-23), 46.00 (C-24), 29.31 (C-25), 19.97 (C-26), 19.55 (C-27), 23.22 (C-28), 12.14 (C-29).

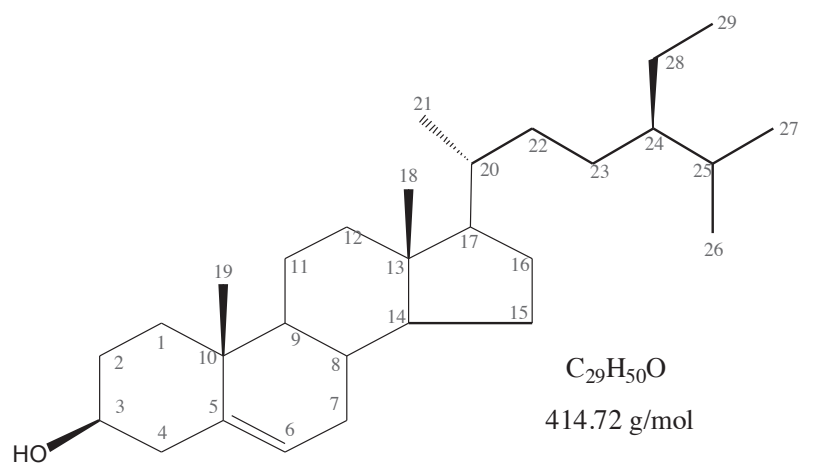

Estructura química de $\boldsymbol{\beta}$-Sitosterol (4)

Vomifoliol (5) con pf $110-111^{\circ} \mathrm{C}$ (lit. 107-109 ${ }^{\circ} \mathrm{C}$ [8]); Rf (Hex:AcOEt:MeOH 4:5:1) = 0.47; EM: $\mathrm{m} / z(\%)$ 225(1), 206(4), 168(9), 150(9), 135(8), 124(100), 122(7), $111(6), 79(6), 69(4) ; \mathrm{RMN}-{ }^{-} \mathrm{H}(300 \mathrm{MHz}$, $\mathrm{CDCl} 3), \delta 5.90(1 \mathrm{H}, \mathrm{m}, \mathrm{H}-5), 5.81(2 \mathrm{H}, \mathrm{m}, \mathrm{H}-7, \mathrm{H}-8)$, $4.41(1 \mathrm{H}, \mathrm{m}, \mathrm{H}-9), 2.45(1 \mathrm{H}, \mathrm{dd}, \mathrm{J}=0.9,17.1, \mathrm{H}-3 \mathrm{a})$, $2.24(1 \mathrm{H}, \mathrm{dd}, \mathrm{J}=1.2,17.1, \mathrm{H}-3 \mathrm{~b}), 1.89(3 \mathrm{H}, \mathrm{d}, \mathrm{J}=1.5$, $\mathrm{H}-13), 1.67(2 \mathrm{H}, \mathrm{sa},-\mathrm{OH}), 1.30(3 \mathrm{H}, \mathrm{d}, \mathrm{J}=6.3, \mathrm{H}-10)$, $1.09(3 \mathrm{H}, \mathrm{s}, \mathrm{H}-11), 1.02(3 \mathrm{H}, \mathrm{s}, \mathrm{H}-12) ; \mathrm{RMN}-{ }^{13} \mathrm{C}(75$ $\mathrm{MHz}, \mathrm{CDCl} 3$ ) $\delta c 79.05$ (C-1), 41.15 (C-2), 49.73 (C-3), 197.81 (C-4), 126.97 (C-5), 162.51 (C-6), 129.01 (C-7), 135.75 (C-8), 68.04 (C-9), 23.78 (C10), 24.05 (C-11), 22.91 (C-12), 18.85 (C-13).

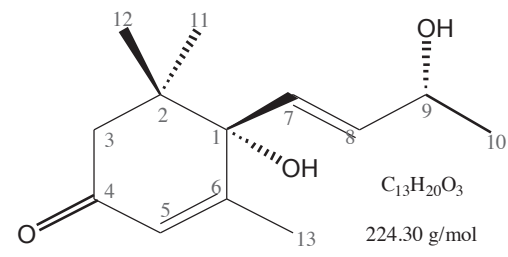

Estructura química de Vomifoliol (5)

\section{Resultados y discusión}

\subsection{Análisis de la fase orgánica (diclorometano)}

De la partición líquido-líquido a partir del extracto metanólico, se obtuvieron dos porciones. La porción soluble en diclorometano, originó 342 eluatos de 50 $\mathrm{mL}$, reunidos según su similitud cromatográfica desarrollada en placas de CCF, en fracciones nombradas en orden alfabético, a partir de los cuales se lograron aislar los compuestos (1), (2), (3), (4) y (5).

El compuesto (1) se purificó de las fracciones J y K como sólido cristalino, con un rendimiento de $0.1 \%$, con las siguientes propiedades: los espectros de RMN de hidrógeno y de carbono-13, indicaron un compuesto de tipo esteroidal, con señales que resuenan en $\delta$ 0.7-2.35, del espectro de $R M N-{ }^{\prime} H$. Las señales de campo alto en el espectro de RMN-1 $\mathrm{H}^{-}(\delta<1)$ fueron asignados a Me-18 $(\delta=0.71, s, 3 H), M e-19(\delta=1.18, s, 3 H), M e-21$ ( $\delta$ $=0.92, d, 3 H)$. Además, se presentó una señal singulete en $\delta 5.72(1 \mathrm{H})$, coherente con la presencia de un protón vinílico. Se puede ver que la muestra no estaba completamente pura, debido a la aparición de señales a $\delta 3.5$ que podrían ser atribuidos a un grupo hidroxilo (3-OH) y $\delta 5.1$ atribuido a protones vinílicos en la cadena lateral; con áreas de pico demasiado pequeñas para considerarse como parte de la molécula. Desde el espectro $\mathrm{RMN}-{ }^{13} \mathrm{C}$, fue posible asignar las resonancias que se refieren a un sistema de carbonilo $a, \beta$-insaturado en $\delta c 199.85$ (C-3), 123.90 (C-4), 171.90 (C-5), respectivamente, de la ciclohexanona. Las asignaciones de estas señales estaban de acuerdo con la literatura [11, $12,5]$. Mientras que el espectro de masas de sitostenona, mostró el pico de ion molecular $\mathrm{M}^{+}$en $\mathrm{m} / \mathrm{z} 412$, y otros iones de fragmentación $\mathrm{m} / \mathrm{z}$ en $397,370,288$, $271,229,187,173,147$ y 124 ; lo que es compatible con la composición molecular de $\mathrm{C}_{29} \mathrm{H}_{48} \mathrm{O}$. Esto fue confirmado por el análisis de los espectros DEPT, que mostraron seis grupos metilo, once grupos de metileno, ocho grupos metino y en la estructura química se observan cuatro átomos de carbono cuaternarios. Basado en análisis detallado y la comparación de los datos espectrales obtenidos experimentalmente con los de la literatura, la estructura correspondió con estigmast-4-en-3-ona, también conocido como estigmastenona [13]. Este compuesto ha sido informado con un punto de fusión de 97-99 ${ }^{\circ} \mathrm{C}[5]$.

El compuesto $2\left[\mathrm{C}_{30} \mathrm{H}_{50} \mathrm{O},\left(\mathrm{M}^{+}\right) 426\right]$ exhibió en los espectros de RMN ${ }^{1} \mathrm{Hy}^{13} \mathrm{C}$ características de lup-20(29)- 
en-3ß-ol: $\delta 4.69$ (m), H-29a; $\delta 4.57$ (m), H-29b; $\delta$ 3.19 (dd, J= Hz 11.2 y 5.0), H-3; $\delta c 79.14, \mathrm{C}-3 ; \delta c$ 151.11, C-20; y ठc 109.48, C-29; y señales debidas a siete grupos de metilo terciario, que también coinciden con el esqueleto base de un triterpeno de tipo lupano, la estructura fue confirmada por comparación de datos espectroscópicos de este compuesto con las descritas en la literatura para lupeol y por su espectro de masas, que muestra fragmentos de iones característicos en $\mathrm{m} / \mathrm{z} 426 \mathrm{M}^{+}, 218,207$ y 189. Además, en el espectro DEPT-135 se distinguen 24 picos $\left(\mathrm{CH}_{3}\right.$, $\mathrm{CH}_{2}, \mathrm{CH}$ ); de los cuales: once corresponden a carbonos de metileno $\left(\mathrm{CH}_{2}\right)$, y según el espectro DEPT-90, seis corresponden a carbonos de metino $(\mathrm{CH})$; por lo tanto, siete son señales debidas a grupos metilo $\left(\mathrm{CH}_{3}\right)$, siendo seis carbonos cuaternarios de acuerdo con la estructura molecular de lupeol.

En las fracciones $\mathbf{J}$ y $\mathbf{K}$, se evidenció la presencia de un ácido graso (3) mediante su espectro de $R M N{ }^{1} H$, en el cual se observa un pico intenso, amplio en aproximadamente $\delta 1.26$ debido a metilenos alifáticos, y señales más pequeñas alrededor $\delta 5.3$ de metinos olefínicos y en $\delta 0.86$ de grupo metilo terminal, en concordancia con las características espectroscópicas reportadas para compuestos lipídicos [9]. Mientras

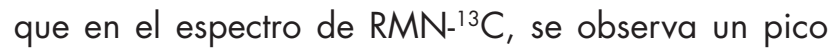
intenso en $\delta c 29.79$ atribuido a grupos $\mathrm{CH}_{2}$ [10].

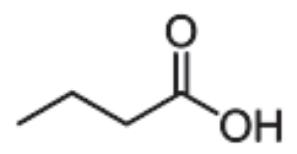

Estructura química general de un ácido graso (3)

El compuesto 4 se purificó como cristales a partir de la fracción $\mathbf{M}$, obteniéndose un rendimiento de $0.3 \%$ en peso. La separación cromatográfica del extracto metanólico de $S$. lateriflorum llevó al aislamiento del compuesto $\beta$-sitosterol por re-cristalización, el cual fue obtenido como un sólido cristalino, con un intervalo de fusión determinado en $128-131^{\circ} \mathrm{C}$ (lit., pf $130-134^{\circ} \mathrm{C}$ [7]). Se compararon los desplazamientos químicos obtenidos con los datos informados para confirmar la identidad de la sustancia. En el espectro de RMN${ }^{1} \mathrm{H}$, se observaron señales típicas de los fitoesteroles, como lo son las señales singuletes de dos grupos metilos angulares de las posiciones $\mathrm{C}-18$ y C-19, y se observó el doblete del grupo metilo de posición C-21.
El característico doblete ancho a $\delta 5.35$ muestra un protón olefínico $(\mathrm{H}-6)$. El espectro de $\mathrm{RMN}-{ }^{13} \mathrm{C}$ mostró 29 señales, que permitieron confirmar la estructura analizada como $\beta$-sitosterol (estigmast-5-en-3-ol), por interpretación del análisis espectroscópico, incluyendo EM y RMN-' $\mathrm{H}$. En el espectro DEPT- 135 se observan 26 señales de grupos $\mathrm{CH}_{3}, \mathrm{CH}$ y $\mathrm{CH} 2$, de los cuales once corresponden a grupos metileno $\left(\mathrm{CH}_{2}\right)$; según el espectro DEPT- 90, nueve picos pertenecen a grupos metino $(\mathrm{CH})$, por lo tanto los seis picos restantes se deben a los metilos $\left(\mathrm{CH}_{3}\right)$. Que junto con el carbono cuaternario de la posición C-5, concuerdan con la fórmula molecular $\mathrm{C}_{29} \mathrm{H}_{50} \mathrm{O}$.

El compuesto 5 fue identificado de las fracciones $\mathbf{A E}$ y $\mathbf{A F}$, por comparación de sus datos espectrales con los reportados en la literatura como vomifoliol, también conocido como Blumenol A. El espectro de masas de vomifoliol mostró el pico de ion molecular en $\mathrm{m} / \mathrm{z} 224$ correspondiente a la fórmula $\mathrm{C}_{13} \mathrm{H}_{20} \mathrm{O}_{3}$. La fórmula molecular sugirió cuatro insaturaciones en la molécula, dos de los cuales estaban justificadas por el sistema carbonilo a, $\beta$-insaturado y se manifiesta claramente por los espectros RMN. Los datos espectrales del compuesto aislado en el presente trabajo fueron compatibles con la estructura de vomifoliol para 5. Así, el espectro de RMN-' ${ }^{-} \mathrm{H}$ de vomifoliol muestra un protón en $\delta 5.90(\mathrm{H}$ $\mathrm{C}(5)$; $\delta \mathrm{c} 126.97)$ y tres protones en $\delta 1.89(\mathrm{Me}-\mathrm{C}(6)$; $\delta c 18.85)$ se mostraron para el grupo Me vinílico situado en el $C(\beta)$ del sistema de carbonilo a, $\beta$-insaturado. Además, estas observaciones junto con los dos dobles de dobles que integra cada una para un protón en $\delta$ $2.45 \mathrm{Ha}-\mathrm{C}(3)$ y $2.24 \mathrm{Hb}-\mathrm{C}(3)$, sugieren un grupo $\mathrm{CH}_{2}$ ubicado adyacente al grupo carbonilo y conectado a un átomo de carbono tetrasustituido. El espectro de RMN ${ }^{-} \mathrm{H}$ mostró señales en $\delta 5.81$ que representan un enlace $C \otimes C$ en una cadena abierta con configuración (E). Se observó también un multiplete que integra para un protón en $\delta 4.41$ (H-C(9); $\delta c$ 68.04). Además, el espectro de $\mathrm{RMN}$ de ${ }^{1} \mathrm{H}$ tiene dos señales de tres protones cada una en $\delta 1.02$ y 1.09 , otra señal de tres protones en $\delta 1.30(\mathrm{~J}=6.3 \mathrm{~Hz}, \mathrm{Me}(10))$.

\subsection{Análisis de la fase hidro-alcohólica}

La fase hidroalcohólica de la partición, después de procederse a acetilar un gramo para mejorar la separación de sus componentes, originó 178 eluatos de $50 \mathrm{~mL}$, a partir de los cuales no se obtuvieron sustancias con perfiles cromatográficos aislables mediante los métodos empleados. 


\section{Conclusiones}

Los metabolitos aislados se identificaron y caracterizaron mediante métodos espectroscópicos, espectrométricos y por la comparación de sus propiedades físicas con las informadas en la literatura. Los compuestos obtenidos del análisis de la fase orgánica del extracto metanólico de las partes aéreas (ramas y hojas) de Sapium lateriflorum fueron cinco substancias las cuales incluyen un ácido graso y cuatro terpenoides. Del análisis de la porción acetilada de la fase hidro-alcohólica del extracto metanólico de S. lateriflorum, mediante cromatografía en columna, no se encontraron compuestos con un perfil cromatográfico adecuado para proceder a separarlos. Los terpenoides son los metabolitos secundarios presentes mayoritariamente en esta especie, presumiblemente, responsables de la actividad biológica.

\section{Agradecimientos}

La presente investigación no hubiera sido posible sin el apoyo del Dr. Fernando Novillo, de la Facultad de Ciencias Químicas de la Universidad Central del Ecuador y del Dr. Guillermo Delgado, del Instituto de Química de la Universidad Nacional Autónoma de México.

\section{Referencias bobliográficas}

1 Chumkaew P., Karalai C., Ponglimanont C. and Chantrapromma K. (2003); Antimycobacterial Activity of Phorbol Esters from the Fruits of Sapium indicum; Journal of Natural Products, 66, 540-543.

2 El-Mekkawy S., Meselhy M., Nakamura N., Hattori M., Kawahata T. and Otake T. (2000); Anti-HIV-1 phorbol esters from the seeds of Croton tiglium; Phytochemistry, 53, 457-464.

3 Caporale L. H. (1995); Chemical ecology view from the pharmaceutical industry; Proceedings of the National Academy of Science of the United States of America, 92, 7582.

4 Murphy E. M., Nahar L., Byres M., Mohammad Shoeb, Siakalima M., Rahman M. M., Gray A. I., Sarker S. D. (2004); Coumarins from the seeds of Angelica sylvestris (Apiaceae) and their distribution within the genus Angelica; Biochemical Systematics and Ecology, 32, 203-207.

5 Prachayasittikul S., Suphapong, S., Worachartcheewan A., Lawung R., Ruchirawat S., and Prachayasittikul V. (2009); Bioactive Metabolites from Spilanthes acmella Murr.; Molecules, 14, 850-867; doi:10.3390/molecules14020850

6 Aynilian G. H., Farnsworth N. R., Persinos G. J. (1972); Isolation of lupeol from Cataeva benthamii; Journal of Phytochemistry, 11, $2885-2886$.

7 McCarthy F. O., Chopra J., Ford A., Hogan S. A., Kerry J. P., O’Brien N. M., Ryan E. and Maguire A. R. (2005); Synthesis, isolation and

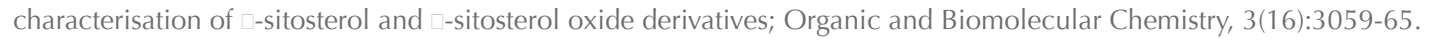

8 Yamano Y. and Ito M. (2005); Synthesis of Optically Active Vomifoliol and Roseoside Stereoisomers; Chemical and Pharmaceutical Bulletin, 53(5) 541-546.

9 Su B.-N., Cuendet M., Farnsworth N. R., Fong H. H. S., Pezzuto J. M., and Kinghorn, A. D. (2002); Activity-guided fractionation of the seeds of Ziziphus jujuba using a cyclooxygenase-2 inhibitory assay; Planta Medica, 68, 1125-1128.

10 Gottlieb H. E., Kotlyar V., and Nudelman A. (1997); NMR Chemical Shifts of Common Laboratory Solvents as Trace Impurities; Journal of Organic Chemistry, 62, 7512-7515.

11 Jamaluddin F., Mohamed S. and Lajis Md. N. (1995); Hypoglycaemic effect of Stigmast-4-en-3-one, from Parkia speciosa empty pods; Food Chemistry, 54, 9-I 3

12 Seca A., Silva A., Silvestre A., Cavaleiro J., Domingues F. and Neto C. (2000); Chemical Composition of the Light Petroleum Extract of Hibiscus cannabinus Bark and Core; Phytochemical Analysis, 11: 345-350

13 Akihisa T, Kimura Y, Roy K, Ghosh P, Thakur S, and Tamura T. (1994); Triterpene alcohols and 3-oxo steroids of nine Leguminosae seeds. Phytochemistry 35: 1309-1313. 